\title{
Wind-driven barotropic gyre I: Circulation control by eddy vorticity fluxes to an enhanced removal region
}

\author{
by Baylor Fox-Kemper ${ }^{1,2}$ and Joseph Pedlosky ${ }^{3}$
}

\begin{abstract}
It is well known that the barotropic, wind-driven, single-gyre ocean model reaches an inertiallydominated equilibrium with unrealistic circulation strength when the explicit viscosity is reduced to realistically low values. It is shown here that the overall circulation strength can be controlled nonlocally by retaining thin regions of enhanced viscosity parameterizing the effects of increased mixing and topographic interaction near the boundaries. The control is possible even when the inertial boundary layer width is larger than the enhanced viscosity region, as eddy fluxes of vorticity from the interior transport vorticity across the mean streamlines of the inertial boundary current to the frictional region. In relatively inviscid calculations the eddies are the major means of flux across interior mean streamlines.
\end{abstract}

\section{Introduction}

It remains unclear what controls the strength of the wind-driven ocean circulation. Many past models of the ocean circulation have relied on an interior solution obeying the Sverdrup (1947) solution. This solution is attached to a western boundary current which is required to close the flow of mass and remove the input of vorticity and energy from the wind. The Sverdrup interior sets the mass flux, and the western boundary current plays a passive role.

While the assumption of a linear, noninertial Sverdrup (1947) interior gives a circulation of roughly the correct strength, the important issue of why a linear vorticity balance is present in the interior remains. Ultimately, there must be a balance between forcing and dissipation. The wind is a constant source of energy and vorticity to the ocean, so without removal of vorticity and energy there is no mechanism for equilibration. However, since the removal is presumed a priori in models with a Sverdrup interior, these models form only a partial understanding of what controls the circulation strength.

Veronis (1966) demonstrates that as vorticity advection becomes strong relative to the

1. MIT/WHOI Joint Program in Oceanography and Ocean Engineering, Woods Hole, Massachusetts, 02543 , U.S.A.

2. Present address: Program in Atmospheric and Oceanic Sciences, Princeton University, Princeton, New Jersey, 08544,U.S.A. email: baylor@princeton.edu

3. Department of Physical Oceanography, Woods Hole Oceanographic Institution, Woods Hole, Massachusetts, 02543, U.S.A. 
frictional removal of vorticity, the inertial terms become dominant in regions outside the boundary current. More recent work has demonstrated that these inertially-dominated or inertial runaway solutions are ubiquitous in the wind-driven single-gyre ocean model with constant viscosity; time-dependent and steady-state calculations with differing boundary conditions all demonstrate this behavior (e.g., Ierley and Sheremet, 1995; Kamenkovich et al., 1995; Sheremet et al., 1995, 1997). These inertially-dominated equilibria occur also in calculations with baroclinic instability (Holland and Lin, 1975) and calculations with multiple gyres (Fox-Kemper, 2004b) at slightly higher Reynolds number than in the single-gyre barotropic model. These inertially-dominated solutions occur at modest inertia to friction ratios, leading one to wonder what occurs in the ocean where the friction is probably much weaker than inertia, yet the ocean is strongly western-intensified with inertial western boundary currents and a relatively noninertial interior flow.

The ratio of inertial terms to friction terms can be considered as a comparison of the inertial boundary layer width (Charney, 1955) to the frictional boundary layer width (Munk, 1950; Stommel, 1948). When the inertial boundary current is significantly wider than the frictional, Il'in and Kamenkovich (1964) and Ierley and Ruehr (1986) demonstrate that a steady western boundary layer structure is no longer possible in regions of outflow from the boundary layer. If viscosity is decreased until the frictional boundary layer becomes thinner than the inertial boundary layer width, the closed innermost streamlines of the time-mean flow do not enter the frictional boundary layer and cannot release the vorticity input within them (as pointed out by Niiler, 1966). Thus, the vorticity within the innermost streamlines - and the circulation around them-will increase dramatically. As the circulation increases, so does the width of the inertial boundary layer. Only when the basin is filled with an inertial recirculation gyre will the frictional terms become large enough to remove the vorticity and energy input.

If bottom pressure torques collude in the removal of vorticity, a similar effect should occur where the inertial boundary current is wider than the region of sloping topography. In a stratified ocean, this effect is even more likely, for the strongest circulation is in the surface layers, and they may only interact with bottom topography near the basin margins or indirectly as in Holland and Rhines (1980). The vorticity budget resulting from the Holland and Rhines (1980) fluxes seems likely to be obtuse, as Haynes and McIntyre (1987) prove that no potential vorticity can be exchanged between isopycnal layers away from the boundaries. Again, the interior streamlines would be isolated from the region of vorticity removal and would increase in strength, which in turn would widen the inertial boundary current and exacerbate the problem. This inertial current formed would then strongly advect vorticity meridionally and violate the primary assumption of the vorticity removal by bottom torque scheme of Hughes and de Cuevas (2001): that the vorticity is removed in a latitude band near where it is input.

The viscosity in numerical models is not intended to represent the action of molecular viscosity; it is many orders of magnitude too large. Obviously the friction used is a parameterization of unresolved processes. In particular, in the traditional flat-bottom models, the friction serves two roles. First, it represents the effects of eddies, as it diffuses vorticity in the interior 
and acts to remove the enstrophy at the smallest scales. Second, it is the only mechanism in these models for the ultimate removal of vorticity from the basin. Thus, it also represents all of the processes which remove vorticity from the basin at the boundary, including both directly frictional and "inviscid" processes; e.g., bottom topography effects.

The use of a constant viscosity tacitly assumes that all the effects of unresolved phenomena can be treated equally: a strong and unrealistic constraint on the parameterized processes. This assumption is severe in a constant depth model where topographic interactions can play no role.

One should be wary of using constant viscosity even when attempting parameterization of only mesoscale processes: ocean observations of these processes produce extremely diverse results. Observations of relative dispersion of subsurface floats by LaCasce and Bower (2000) indicate approximately an order of magnitude variation in turbulent diffusivity depending on location. This variation in diffusivity is primarily linked to the geographic variation in the strength of mesoscale eddy stirring. On the other hand, the variable viscosity used in this model is intended primarily to parameterize unrepresented physics. Nonetheless, these observations lend credibility to the form of the parameterization and the range of viscosity variation used in the model.

There are additional physical interactions near the boundaries which cannot be included without including additional physics. To verify whether the model friction can be replaced by the action of eddies in the basin interior but not at the boundaries, the approach used here is to try to treat these boundary phenomena simply and see the result on the overall circulation. It will be shown here that as the viscosity is lowered in the basin interior, so long as a sufficiently strong mechanism for vorticity removal exists near the boundary the mean flow remains western-intensified and is controlled by an eddy flux to the region of the enhanced vorticity removal.

This paper is organized in sections. In Section 2, the specifics of the model used are presented. Section 3 presents diagnostic and analytic constructs that are used in understanding the behavior of the model. In Section 4 the results of the numerical calculations are presented.

\section{Boundary-enhanced viscosity in the homogeneous model}

The model used here is the rigid lid, homogeneous density, single-gyre ocean model on a $\beta$-plane with spatially-varying viscosity. Because of its relative simplicity, ease of implementation, and the physical plausibility of a proportionality between the flux and the large-scale vorticity gradient, the lateral friction used is a horizontal diffusion of relative vorticity $(\nabla \cdot$ $\delta_{M}^{3} \nabla \zeta$ ) with a spatially-varying horizontal eddy viscosity. The viscosity is scalar and varies only in the zonal direction (i.e., perpendicular to the western boundary) in these calculations.

This form of viscosity was chosen for a number of reasons. First, together with the $\beta$-plane approximation, it guarantees that the friction used is the same as if a diffusion of absolute vorticity were used (as $\nabla \cdot \delta_{M}^{3} \nabla\left(\zeta+\beta y\right.$ ) is the same as $\nabla \cdot \delta_{M}^{3} \nabla \zeta$ when $\delta_{M}^{3}$ is a function of $x$ only and $\beta$ is constant). In this respect, it is a representation of subgrid scale processes which diffusively flux vorticity. Other possible choices of lateral friction operator are available such as a diffusive flux of momentum or the more dubious choice of 
$\delta_{M}^{3} \nabla^{2} \zeta$. However, momentum is not fluxed component by component by eddy processes so the momentum flux parameterization is not used, and the latter form is not used because its flux is not proportional to a gradient of the large-scale flow properties. In this section, a bottom friction also appears in the equations to demonstrate its role, although numerical results with bottom friction are presented elsewhere (Fox-Kemper, 2003, Section 3.7).

The model solves the following dimensionless barotropic vorticity equation (1) and then inverts the Poisson equation (2) to find the streamfunction at each time step:

$$
\begin{gathered}
\frac{\partial \zeta}{\partial t}+\delta_{I}^{2}\left(\frac{\partial \psi}{\partial x} \frac{\partial \zeta}{\partial y}-\frac{\partial \psi}{\partial y} \frac{\partial \zeta}{\partial x}\right)+\frac{\partial \psi}{\partial x}=-\sin (\pi y)+\nabla \cdot \delta_{M}^{3} \nabla \zeta-\delta_{s} \zeta, \\
\zeta=\nabla^{2} \psi .
\end{gathered}
$$

The dimensionless variables are related to dimensional ones in the following way (asterisks denote dimensional variables):

$$
\begin{gathered}
t_{*} \equiv \frac{t}{\beta L}, \quad x_{*} \equiv L x, \quad y_{*} \equiv L y, \\
w_{E *} \equiv-W_{E} \sin (\pi y), \quad \psi_{*} \equiv \frac{W_{E} f_{0} L}{\beta D} \psi, \\
\delta_{I}^{2} \equiv \frac{W_{E} f_{0}}{\beta^{2} D L^{2}}, \quad \delta_{M}^{3} \equiv \frac{A_{H}}{\beta L^{3}}, \quad \delta_{S} \equiv \frac{r}{\beta L} .
\end{gathered}
$$

$A_{H}$ is the Austausch coefficient in the horizontal direction and $r$ is the bottom friction decay rate. $L$ is the horizontal basin dimension. $D$ is the basin depth (or layer depth). The variable $\zeta$ represents relative vorticity, while $\psi$ is the streamfunction of the velocity $[u=$ $-(\partial \psi / \partial y), v=(\partial \psi / \partial x)]$. The streamfunction $\psi$ is scaled so that the Sverdrup solution would have a maximum of $\psi=1$ were it to fill the dimensions of the basin. The $\beta$-plane approximation is used, so the value of the Coriolis parameter at the center of the domain is $f_{0}$ and its meridional derivative is $\beta$.

The single-gyre wind forcing $\left(w_{E}=-\sin (\pi y)\right.$ : a negative input of vorticity throughout the basin) is intended to roughly model a northern hemisphere subtropical gyre. In the double-gyre model, as used by Marshall (1984), for example, there is no net input of vorticity, so purely internal mechanisms might remove the vorticity. The importance of adding a second gyre is discussed in Fox-Kemper (2004b). In the real ocean, there is net vorticity input, and the removal of this vorticity is the oceanic phenomenon to be studied here so the single-gyre model is preferable initially.

The boundaries are located at $x=0$ and $x=1$ in the zonal direction and at $y=0$ and $y=1$ in the meridional direction. The boundaries are impermeable, which is implemented by setting $\psi=0$ for solutions of (2), an appropriate method for a constant depth model.

The lateral friction in (1) also requires higher-order boundary conditions. The eastern and western boundaries have the no-slip boundary condition $[(\partial \psi / \partial x)=0]$ for "solid" 
boundaries, while the other boundaries have slip $(\zeta=0)$ for "fluid" boundaries. The no-slip boundary condition effectively generates instabilities at the western boundary, and the slip boundary condition at the north allows ready comparison with double-gyre calculations.

Of course, the boundary conditions critically affect the solutions and especially the dynamics of the frictional sublayer. Calculations with slip boundary conditions at all boundaries were also performed (Fox-Kemper, 2003, Section 3.8). The mechanism proposed here requires a rich eddy field, which is present in no-slip models due to shear instability and in multi-layer models with any boundary condition due to baroclinic instability. The presence of a strong eddy field in ocean observations is indisputable. The weaker shear near the boundary in barotropic slip calculations produces few eddies near the boundary; thus the barotropic slip model is exceptional and produces misleading results. Even so, the circulation control detailed here is present even with the weaker eddy field found in slip calculations, but the no-slip calculations are more illustrative. Super-slip boundary conditions are inappropriate for any model which requires vorticity removal by lateral friction for equilibration, as they allow for no vorticity flux through the boundaries at all.

The parameters, $\delta_{I}, \delta_{M}$, and $\delta_{S}$, would be the Charney (1955), Munk (1950), and Stommel (1948) boundary layer scales, respectively, if $\delta_{M}$ were constant in a basin of unit width. The viscosity parameter, $\delta_{M}^{3}$, is proportional to the dimensional viscosity and plays a similar role in the nondimensional equations but has different units.

For the calculations presented here, the value of $\delta_{I}$ is fixed at 0.02 . The value of $\delta_{M}$ varies across the different calculations and throughout the basin. The Reynolds number of the boundary layer for a given viscosity is a useful measure for comparing different runs; it is defined here as $\delta_{I}^{3} / \delta_{M}^{3}$ (other authors may use the Reynolds number of the basin-wide flow, which is $\left.\delta_{I}^{2} / \delta_{M}^{3}\right)$.

The viscosity parameter varies smoothly between an interior and a boundary value (near the "solid" boundaries):

$$
\begin{gathered}
\delta_{M}^{3}=\frac{\delta_{I}^{3}}{\operatorname{Re}_{\mathrm{i}}}+\left(\frac{\delta_{I}^{3}}{\operatorname{Re}_{\mathrm{b}}}-\frac{\delta_{I}^{3}}{\operatorname{Re}_{\mathrm{i}}}\right)\left(e^{-x / \delta_{d}}+e^{-(1-x) / \delta_{d}}\right), \\
\delta_{d} \equiv \frac{\delta_{I}}{\sqrt{\operatorname{Re}_{\mathrm{i}}}} .
\end{gathered}
$$

The parameters $\mathrm{Re}_{\mathrm{b}}$ and $\mathrm{Re}_{\mathrm{i}}$ are fixed. Thus, the viscosity parameter decays exponentially from the boundary value to the interior value with a decay scale equal to the frictional sublayer scale, $\delta_{d}$. This functional form was chosen for simplicity and smoothness, rather than from any particular parameterization of a physical process.

The frictional sublayer scale is found by hypothesizing a balance between the mean advection and the frictional terms (e.g., Pedlosky, 1996). One advantage of choosing the frictional sublayer scale as the decay scale for the viscosity parameter is that the width of the frictional sublayer will be relatively unchanged by a change in $\mathrm{Re}_{\mathrm{b}}$. The second advantage is that when $\operatorname{Re}_{\mathrm{i}}$ is greater than one, the inertial boundary layer scale will be larger than the 


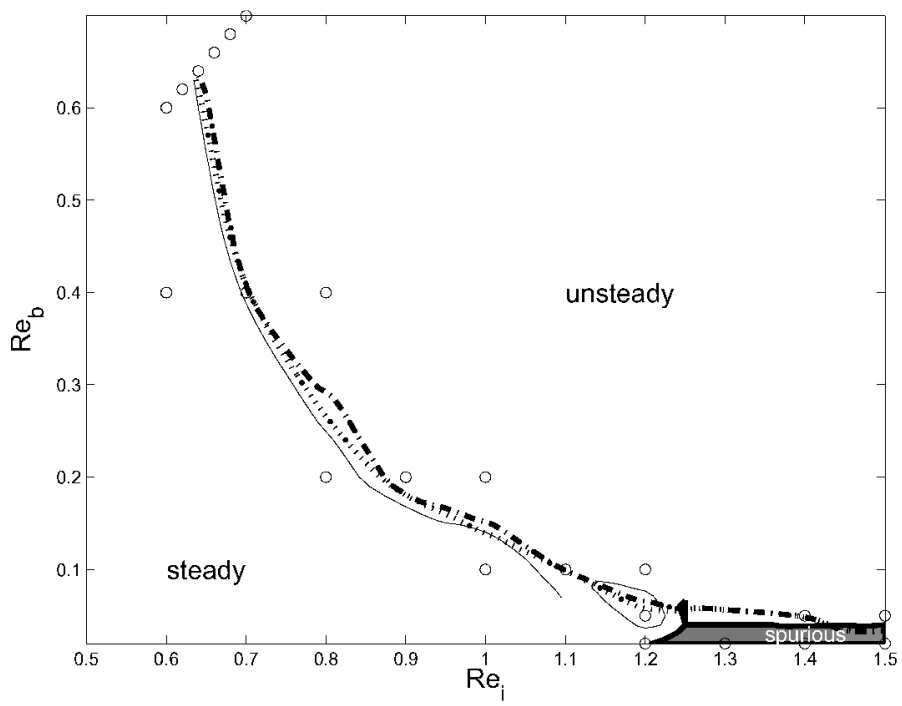

Figure 1. Estimate of the onset of instability for calculations with $\delta_{I}=0.02$ from interpolation of energy growth rate after spin-up in calculations (indicated with dots) at different resolutions: $33 \times$ 33 (solid), $65 \times 65$ (dotted), and $129 \times 129$ polynomials (dash-dotted). Spurious instabilities grow rapidly in the shaded region, but only at $33 \times 33$ resolution.

frictional sublayer. Therefore, as $\mathrm{Re}_{\mathrm{i}}$ becomes greater than one, fewer of the mean streamlines will pass through the enhanced viscosity, and relatively inviscid dynamics must occur along these streamlines. This decay scale also emphasizes that decreasing $\mathrm{Re}_{\mathrm{b}}$ only raises the viscosity within a region which was already strongly influenced by friction.

A Chebyshev pseudo-spectral model was created to solve (1) and (2) with the viscosity parameter given by (3) (Fox-Kemper, 2003, Appendices A and C). A Chebyshev polynomial model was chosen because Gottlieb and Orszag (1981) report these models to be resistant to the artificial instabilities common to models using variable diffusivities. This is confirmed by repeated calculations varying spatial and temporal resolution that result in very similar time-mean fields (Fox-Kemper, 2003, Appendix C). Also, Figure 1 demonstrates that the onset of instability in this model is rather insensitive to resolution with $\mathrm{Re}_{\mathrm{b}}$ and $\mathrm{Re}_{\mathrm{i}}$ separated by more than an order of magnitude. Rather than producing additional instabilities by numerical issues of viscosity gradients, decreasing $\mathrm{Re}_{\mathrm{b}}$ with fixed $\mathrm{Re}_{\mathrm{i}}$ stabilizes the flow, as one would expect when making a flow more viscous. The spurious instability is easily controlled with sufficient resolution $(257 \times 257$ modes are used for all subsequent calculations).

The range of $\delta_{I}$ and $\delta_{M}$ used is governed by both numerical constraints and an attempt to reach the correct parameter range. The value of $\delta_{I}$ is 0.02 in all of the calculations presented here. The maximum interior Reynolds number obtainable with reasonable accuracy of evaluation of the frictional terms at the resolution used $(257 \times 257$ polynomials) was 9 for this value of $\delta_{I}$. The accuracy at this resolution was confirmed by spectral decay of the Chebyshev 
coefficients of relative vorticity for all parameter settings, and by comparison with higher and lower resolution calculations for some parameter settings. Although the runs at different resolutions differed in the details of the eddy field, the time-mean circulation and magnitude of the kinetic energies agreed.

The value of $\delta_{I}$ is physically relevant and lies in the range used in similar calculations. Ierley and Sheremet (1995) use values surrounding 0.02, Kamenkovich et al. (1995) use 0.01, and Bryan (1963) advocates a range of values from 0.03 to 0.005 depending on the depth of the moving layer and basin dimension. The value of 0.02 used here corresponds to a $80 \mathrm{~km}$ inertial boundary current scale in a $4,000 \mathrm{~km}$ basin with a velocity scale of $0.1 \mathrm{~ms}^{-1}$ (with $\beta=2 \cdot 10^{-11} \mathrm{~m}^{-1} \mathrm{~s}^{-1}$ ). The time units $(\beta L)^{-1}$ correspond to about 3.5 hours, and a typical integration duration of 10,000 units is approximately four years.

Although proper interpretation of eddy viscosity is model dependent, and it is therefore inherently difficult to measure, the value of $\delta_{M}$ used here is probably too large. The maximum value of the Reynolds number here is 9 , while estimates of a more appropriate eddy viscosity place the appropriate Reynolds number in the range of 10-1000 (Pedlosky, 1987). Results in Section 4a indicate that the circulation can be controlled easily with a modest change in $\mathrm{Re}_{\mathrm{b}}$ for all $\mathrm{Re}_{\mathrm{i}}$ used, and the results in Section 4 of the companion paper indicate that this seems likely to continue to much larger $\mathrm{Re}_{\mathrm{i}}$. Even for the parameters used, the value of $\delta_{M}$ is small enough to ensure that the frictional sublayer is clearly smaller than the inertial boundary layer, which is the desired physical criterion.

\section{Analysis}

This section presents some analytic results and diagnostic tools derived from consideration of vorticity budgets.

The barotropic vorticity equation can be written in flux form, where every term is a conservative flux of vorticity. This is also true for the Reynolds-averaged barotropic vorticity equation.

$$
\begin{gathered}
\frac{\partial \zeta}{\partial t}+\nabla \cdot\left(\hat{\mathbf{x}} \psi+\delta_{I}^{2} \mathbf{u} \zeta-\delta_{M}^{3} \nabla \zeta+\delta_{S} \nabla \psi\right)=-\sin (\pi y), \\
\frac{\partial \bar{\zeta}}{\partial t}+\nabla \cdot\left(\hat{\mathbf{x}} \bar{\psi}+\delta_{I}^{2} \overline{\mathbf{u}} \bar{\zeta}+\delta_{I}^{2} \overline{\mathbf{u}^{\prime} \zeta^{\prime}}-\delta_{M}^{3} \nabla \bar{\zeta}+\delta_{S} \nabla \bar{\psi}\right)=-\sin (\pi y) .
\end{gathered}
$$

The overbar denotes a time-mean over a long time-scale compared to the eddy time scales, and primes denote fluctuations from the mean. Throughout this paper, the time-mean will be taken over the second half of the model calculation, and the definition of the eddy field will be the deviation from such a time mean. It is useful to keep in mind a third time scale, one which is long compared to the eddies, but short enough to vary during, for example, the spin-up time of the model. On these time scales, the dominant effects are the pooling and draining of vorticity in different regions of the basin.

Every term in (6) is a flux of vorticity except the wind vorticity source and the rate of 
change term, although these terms can also be written as fluxes if helpful. Because all of the terms are fluxes, the vorticity input by the wind cannot be locally removed. Haynes and McIntyre (1987) and Marshall and Nurser (1992) point out that a flux form also results for the fluid between isopycnal layers. In this sense the barotropic model used here is an initial step toward understanding the vorticity removal in a three-dimensional model.

The Reynolds-averaged vorticity fluxes that will be discussed are the $\beta$-flux $(\bar{\psi} \hat{\mathbf{x}})$, the mean flux $\left(\delta_{I}^{2} \overline{\bar{u}} \bar{\zeta}\right)$, the eddy flux $\left(\delta_{I}^{2} \overline{u^{\prime} \zeta^{\prime}}\right)$, the bottom friction flux $\left(\delta_{S} \nabla \bar{\psi}\right)$, and the lateral friction flux $\left(-\delta_{M}^{3} \nabla \bar{\zeta}\right)$.

Unlike vorticity, energy can be locally dissipated. However, the work done differs depending on the alignment of the flow with the wind stress, so the vorticity budget is a simpler tool for analysis of this model. Further discussion of the energetics of the model is presented in Fox-Kemper (2003, Section 3.6) and discussion of energetics in a similar model can be found in Scott and Straub (1998).

The integral of the Reynolds-averaged vorticity equation within a mean streamline is useful in understanding the role of eddy and frictional fluxes in this model. If the mean is taken over the entire time interval, then $(\partial \bar{\zeta} / \partial t)$ vanishes. Using the divergence theorem and noting that the mean fluxes cancel out when integrating along a mean streamline, the following results for the area enclosed by the mean streamline $\bar{\psi}_{C}$, denoted by $A\left(\bar{\psi}_{C}\right)$ are:

$$
\oint_{\bar{\psi}_{c}}\left(\delta_{I}^{2} \overline{\mathbf{u}^{\prime} \zeta^{\prime}}+\delta_{S} \nabla \bar{\psi}-\delta_{M}^{3} \nabla \bar{\zeta}\right) \cdot \hat{\mathbf{n}} \mathrm{ds}=-\iint_{A\left(\bar{\psi}_{c}\right)} \sin (\pi y) \mathrm{dxdy}
$$

Thus, the vorticity flux across mean streamlines can only be carried by the friction or by the eddies and must balance the wind input within that streamline.

The vorticity contained within a mean streamline is an important quantity, of course, because the integrated vorticity is directly related to the circulation. In fact, the mean circulation around a mean streamline is the integrated mean vorticity within that streamline. Thus, any imbalance in the vorticity fluxes across a particular streamline will lead to a change in the circulation around that streamline.

If the streamline chosen is the one located at the boundary $\left(\bar{\psi}_{C}=0\right)$, the basin-wide budget is produced.

$$
\oint_{\bar{\psi}_{c=0}}\left(\delta_{S} \nabla \bar{\psi}-\delta_{M}^{3} \nabla \bar{\zeta}\right) \cdot \hat{\mathbf{n}} \mathrm{ds}=-\frac{2}{\pi} .
$$

It is obvious from (8) that it is exceedingly difficult to reduce the viscosity at the boundary without affecting the mean flow in the single-gyre model.

Eq. (8) demonstrates the dynamical reason for emphasis on unresolved boundary processes in these experiments. Regardless of the model used, because the vorticity equation can be written in flux form, there must always be a process at the edges of the domain capable of removing the vorticity. The model chosen here is enhanced viscosity at 


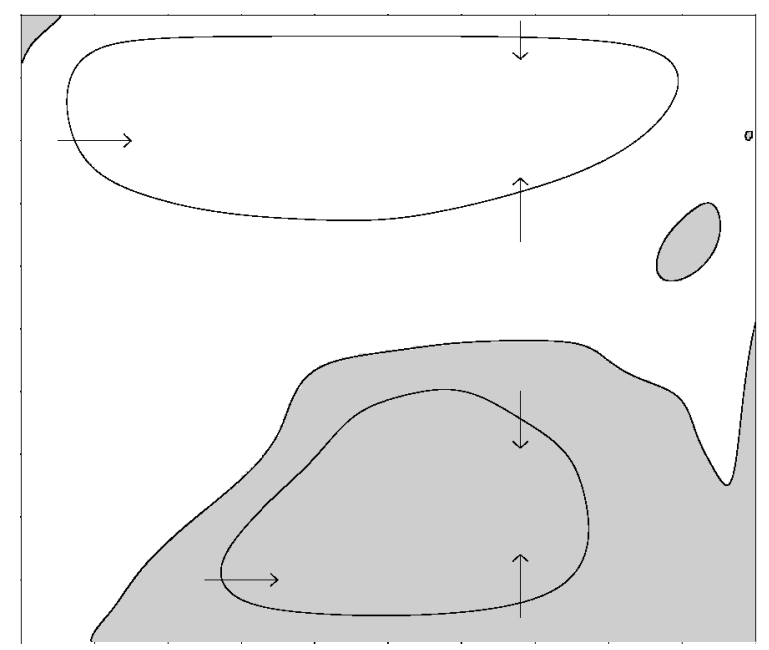

Figure 2. Schematic of regions of integration useful in converting from flux divergences to flux out of a particular region. Arrows denote fluxes, and regions of negative streamfunction are shaded.

the boundary, as (8) is evaluated using the value of the viscosity at the boundary only. Thus, in the region where the eddies are unable to participate, the viscosity is increased so that the mean field gradients need not be as large. By using a large viscosity at the boundary, (8) can be satisfied and by simultaneously using a small interior viscosity (7) can be dominated by resolved eddies rather than by the friction.

If the boundary condition is no-slip, then $\delta_{S} \nabla \bar{\psi}=0$, and neither bottom friction nor eddies can contribute to the basin-wide vorticity budget. For this reason, the bottom friction is not used for most of the calculations presented here $\left(\delta_{S}=0\right)$. Calculations with bottom friction are presented in Fox-Kemper (2003, Section 3.7) and reveal that neglecting bottom friction does not affect the primary implications of this work.

Much of the information contained in the flux field is not relevant to understanding the effects of the eddies on the mean flow. Only the divergences of the fluxes appear in (5), and this is also true of the Reynolds-averaged equation (6). Therefore, in determining the effects of the eddies on the mean flow, it is more important to determine the flux divergences than the fluxes themselves. The Reynolds fluxes tend to be dominated by the largely spiraling nondivergent motion, and so direct examination is not particularly revealing. Furthermore, the Helmholtz decomposition into rotational and divergent fluxes cannot be used reliably as it is not unique in a bounded domain (Fox-Kemper et al., 2003). However, direct examination of eddy buoyancy fluxes in a baroclinic model may be quite revealing (e.g., Berloff, 2003).

Nonetheless, sometimes it is more intuitive to consider fluxes, or at least to connect the divergences via hypothetical fluxes. Figure 2 schematizes integration of the flux divergence field within time-mean streamlines of the flow, as in (7).

In most of the single-gyre calculations, there is a region where the time-mean streamfunc- 
tion is dominantly positive, and possibly a region where the streamfunction is negative. Within the positive region, the streamlines will largely be nested one within another, and likewise for the negative streamlines. Therefore the streamfunction is nearly monotonic, and the two-dimensional space can be reduced to a one-dimensional space with the mean streamfunction as the independent variable. Integration over the area enclosed within a streamline of positive streamfunction is equivalent to integration over the region where the streamfunction exceeds that value. Likewise, integration over the area enclosed within a negative streamfunction streamline amounts to integration over the area where the streamfunction is less than that value. In this way, the fluxes can be determined in the outward normal direction. For example, the average outward normal eddy flux can be determined by

$$
\oint_{\bar{\psi}_{c}}\left(\delta_{I}^{2} \overline{\mathbf{u}^{\prime} \zeta^{\prime}}\right) \cdot \hat{\mathbf{n}} \mathrm{ds}=\left\{\begin{array}{ll}
\iint_{\bar{\psi} \leq \bar{\psi}_{c}} \nabla \cdot\left(\delta_{I}^{2} \overline{\mathbf{u}^{\zeta}}\right) \mathrm{dxdy} & \text { for } \bar{\psi}_{c}<0 \\
\iint_{\bar{\psi} \geq \bar{\psi}_{c}} \nabla \cdot\left(\delta_{I}^{2} \overline{\mathbf{u}^{\zeta}}\right) \mathrm{dxdy} & \text { for } \bar{\psi}_{c}>0
\end{array} .\right.
$$

When the streamfunction is not simple, some complications arise; interpreting the $\bar{\psi}=0$ streamline can be challenging. Because of the impermeability boundary condition, this streamline surrounds the entire basin, but it also divides the regions of positive and negative streamfunction. Thus, three sensible calculations of the total flux across the $\bar{\psi}=0$ streamline exist. First, one could integrate over the whole basin. This would give the flux out of the basin, and it is useful as in (8). Second, one could integrate the divergence over all regions whose streamfunction is greater than zero. In this case, it would be the total flux out of all of the unshaded regions in Figure 2. Third, one could integrate the divergence over all regions whose streamfunction is less than zero.

A lesser problem in interpretation exists when the streamfunction has more than one local maximum or minimum in the interior. Figure 2 shows that these isolated closed streamlines may exist in the mean streamfunction field; they are shown in that figure as small shaded regions. Including these isolated closed streamlines with the larger-areaenclosing streamlines in the integration is the convention here, but their small vorticity flux means inclusion or exclusion makes little quantitative impact.

Despite the fact that there are no net fluxes by the mean flow across mean streamlines, the mean flow still contributes to the fluxes across mean streamlines by rearranging their location. This prevents direct comparison of the fluxes for different calculations with different mean flows. To allow comparison, the vorticity fluxes are used to construct a measure similar to the Nusselt number used in thermal convection. The Nusselt number is a ratio of the total heat fluxes to the nonconvective fluxes used to assess the increase in efficiency of heat transport due to the presence of convection. Here, the analogue is the ratio of the total vorticity transport across a mean streamline to the frictional flux across 
that mean streamline. The eddies in this model are primarily caused by shear instabilities, so like the frictional flux they are also dependent on the vorticity gradient (although their dependence may be nonlocal). Thus, just as with the Nusselt number, if this flux ratio $\left(\mathrm{Nu}_{\zeta}\right)$ is large, the eddies are transporting a large flux compared to the frictional flux and the eddy flux is considered to be efficient. The ratio is defined by

$$
\mathrm{Nu}_{\zeta}\left(\bar{\psi}_{c}\right) \equiv \frac{\oint_{\bar{\psi}_{c}}\left(\delta_{I}^{2} \overline{\mathbf{u}^{\prime} \zeta^{\prime}}+\delta_{S} \nabla \bar{\psi}-\delta_{M}^{3} \nabla \bar{\zeta}\right) \cdot \hat{\mathbf{n}} \mathrm{ds}}{\oint_{\bar{\psi}_{c}}\left(\delta_{S} \nabla \bar{\psi}-\delta_{M}^{3} \nabla \bar{\zeta}\right) \cdot \hat{\mathbf{n}} \mathrm{ds}} .
$$

The Nusselt number is usually defined with a denominator that is the frictional or diffusive flux which would occur in the absence of motion. Here, the denominator of $\mathrm{Nu}_{\zeta}$ depends on the time-mean vorticity gradient which changes throughout the basin and as the parameters change. The flux across the innermost nested streamline is small (it encloses little wind input) while the flux across the streamline closest to the boundary is large (it encloses almost all the wind input). For this reason, $\mathrm{Nu}_{\zeta}$ is a function of the mean streamfunction and not as satisfying as the Nusselt number (and should therefore not be directly compared to convective Nusselt numbers), but it seems the best choice available.

\section{Computational results}

The major computational results found with this model are presented here as subsections. The first subsection shows that increasing the viscosity only within the frictional sublayer controls the overall circulation nonlocally. The second subsection shows that outside the frictional sublayer, the eddy flux of vorticity is responsible for the flux across mean streamlines.

\section{a. Control of circulation strength}

Figure 3 shows the time-mean streamfunction resulting from different values of $\operatorname{Re}_{\mathrm{b}}$ and $\mathrm{Re}_{\mathrm{i}}$. The time means are taken over the second half of the total integration time. For a given $\mathrm{Re}_{\mathrm{i}}$, the circulation strength can be reduced by decreasing $\mathrm{Re}_{\mathrm{b}}$. This effect depends critically on the time dependence of the solution, because much of the mean flow never passes through the region of enhanced viscosity. Therefore, the presence of eddies allows control of the circulation in the interior nonlocally by the frictional sublayer at the boundary.

The ratio of the kinetic energy of the mean flow and the total kinetic energy (which includes both eddy and mean flow energy) changes as $\mathrm{Re}_{\mathrm{i}}$ increases. Figure $4 \mathrm{a}$ shows that the kinetic energy contained in the mean flow is reduced by decreasing $\operatorname{Re}_{\mathrm{b}}$. Figure $4 \mathrm{~b}$ shows that the total kinetic energy continues to increase with increasing $\mathrm{Re}_{\mathrm{i}}$ despite changes to $\mathrm{Re}_{\mathrm{b}}$. The ratio is relatively insensitive to the value of $\mathrm{Re}_{\mathrm{b}}$, so the energy in the eddies is strongly affected by $\operatorname{Re}_{\mathrm{i}}$ but not by $\mathrm{Re}_{\mathrm{b}}$. The energy increase in the eddies with $\mathrm{Re}_{\mathrm{i}}$ occurs regardless of whether or not the mean flow is controlled by $R_{b}$. Thus, it seems that as long as there is a mechanism for ultimate removal of the vorticity, an arbitrary amount of 

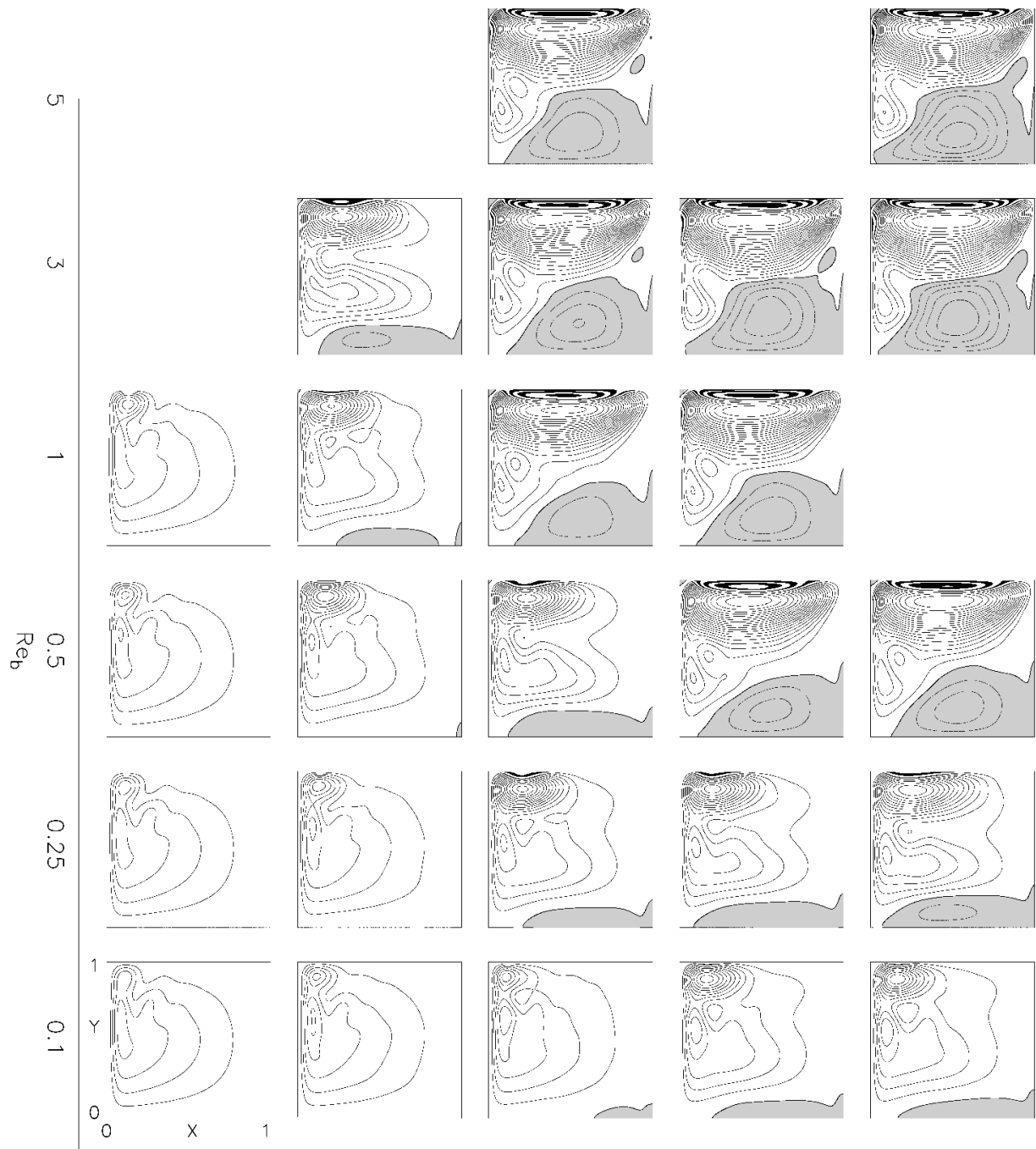

3

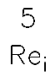

7

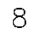

Figure 3. Collage of contours of the time-mean streamfunction for different values of $\operatorname{Re}_{i}$ and $\operatorname{Re}_{b}$. The contour interval is 0.2 in units where 1 is the maximum of the Sverdrup solution. Regions of negative streamfunction are shaded.

energy can be placed in the eddy field by setting the value of $\mathrm{Re}_{\mathrm{i}}$ while control of the mean flow is achieved by setting the value of $\mathrm{Re}_{\mathrm{b}}$.

The inertially-dominated calculations in Figure 4a have been indicated. Once the recirculation gyre reaches the eastern boundary, the parametric change in mean flow kinetic energy with $\mathrm{Re}_{\mathrm{i}}$ changes. In fact, some of the calculations show a decrease with 


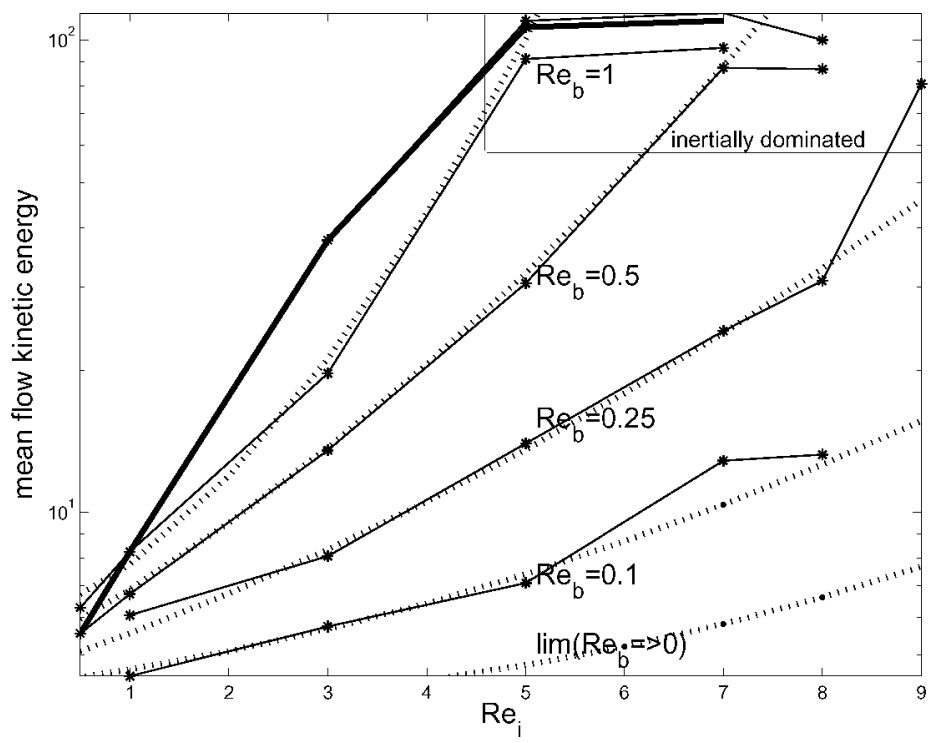

a.

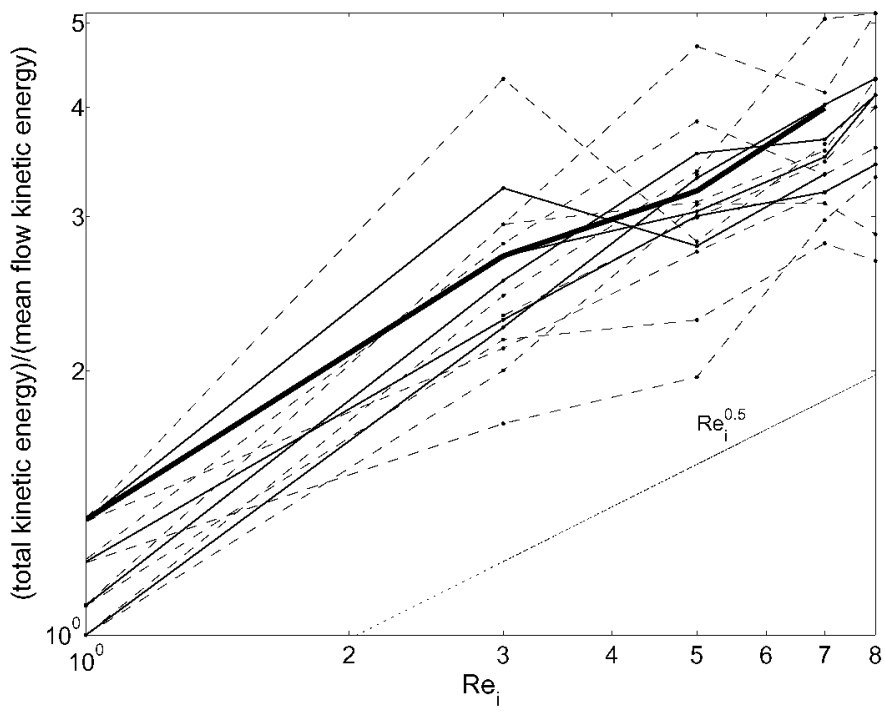

Figure 4. (a) shows the kinetic energy of the time-mean flow from calculations with different $\operatorname{Re}_{\mathrm{b}}$ and $\mathrm{Re}_{\mathrm{i}}$. The thin solid lines connect results with a particular $\mathrm{Re}_{\mathrm{b}}$, and the thick line connects the results where $\mathrm{Re}_{\mathrm{b}}=\mathrm{Re}_{\mathrm{i}}$. The dotted lines are examples of the empirical fit (11). (b) plots the ratio of the mean total kinetic energy to the kinetic energy of the time-mean flow for calculations with different $\mathrm{Re}_{\mathrm{i}}$ and $\mathrm{Re}_{\mathrm{b}}$. The dashed lines show the range of variability during the interval of averaging. A line proportional to $\sqrt{\mathrm{Re}_{\mathrm{i}}}$ is included at lower right. 
increasing $\mathrm{Re}_{\mathrm{i}}$ (while the total kinetic energy continues to increase). This change in parametric response can be traced to the action of eddies around the edge of the recirculation gyre efficiently removing vorticity from the eastern boundary frictional sublayer. Thus, one can define inertial domination by either the size of the recirculation gyre or the parametric dependence. This effect, while interesting in its own right, cannot be important in any western-intensified ocean.

While the mean flow scales more or less exponentially with $\operatorname{Re}_{i}$ for a given value of $\operatorname{Re}_{b}$ until inertial domination sets in, the total energy to mean energy increase scales approximately with $\sqrt{\mathrm{Re}_{\mathrm{i}}}$. Thus, for western intensified calculations, empirical formulae for the energies are:

$$
\begin{gathered}
\iint \frac{\bar{\psi}^{2}}{2} \mathrm{dxdy} \approx \exp \left(\left[\operatorname{Re}_{\mathrm{i}}^{2}, \operatorname{Re}_{\mathrm{i}}, 1\right]\left[\begin{array}{ccc}
0.08 & -0.02 & 0.01 \\
-0.66 & 0.90 & -0.02 \\
-0.23 & 0.58 & 1.41
\end{array}\right]\left[\begin{array}{c}
\mathrm{Re}_{\mathrm{b}}^{2} \\
\mathrm{Re}_{\mathrm{b}} \\
1
\end{array}\right]\right), \\
\iint \frac{\overline{\psi^{2}}}{2} \mathrm{dxdy} \approx \frac{1.36}{\sqrt{\operatorname{Re}_{\mathrm{i}}}} \iint \frac{\bar{\psi}^{2}}{2} \mathrm{dxdy} .
\end{gathered}
$$

The empirical fit is shown in Figure $4 \mathrm{a}$ with dotted lines. Extrapolation to higher $\operatorname{Re}_{\mathrm{i}}$ using these empirical formulae is not likely to be accurate. However, the trend implied by (11) as $\mathrm{Re}_{\mathrm{b}}$ goes to zero is that the mean flow kinetic energy will change very slowly with $\mathrm{Re}_{\mathrm{i}}$. The slow rate of change is indicated by the small magnitude of the first two elements in the third column of the coefficient matrix in (11). As $\mathrm{Re}_{\mathrm{i}}$ increases, the total kinetic energy (and hence the eddy energy) will continue to grow roughly as $\sqrt{\mathrm{Re}_{\mathrm{i}}}$. Thus, circulation control to higher $\operatorname{Re}_{\mathrm{i}}$ is consistent with the behavior observed here.

Moreover, we have chosen to reduce the size of the region for enhanced viscosity with $\operatorname{Re}_{\mathrm{i}}\left[\delta_{d} \equiv\left(\delta_{I} / \sqrt{\mathrm{Re}_{\mathrm{i}}}\right)\right]$. This choice was made to emphasize that the viscosity was being decreased only in a region that was already strongly frictional. This continued decrease in size seems unlikely to appear in any realistic parameterization of the effects of bottom topography, for example. Our choice is a stringent test for the limiting behavior of all such parameterizations in the limit of high $\operatorname{Re}_{\mathrm{i}}$.

Figure 5 shows typical snapshots and averages of the absolute vorticity and streamfunction for an inertially-dominated and a western-intensified calculation with $\operatorname{Re}_{i}=5$. The only difference in parameters between the two calculations is a region of enhanced viscosity less than half as wide as the inertial boundary layer width. The eddy kinetic energy is strongest in the west for the western-intensified equilibrium. In both cases, the eddies interact with the recirculation gyre.

Thus, the time-mean circulation can be controlled by a thin region of enhanced viscosity near the boundary. This region may be thinner than the inertial boundary layer width. Despite control of the mean flow, the total energy and in particular, the eddy energy, continues to increase as $\mathrm{Re}_{\mathrm{i}}$ increases. 


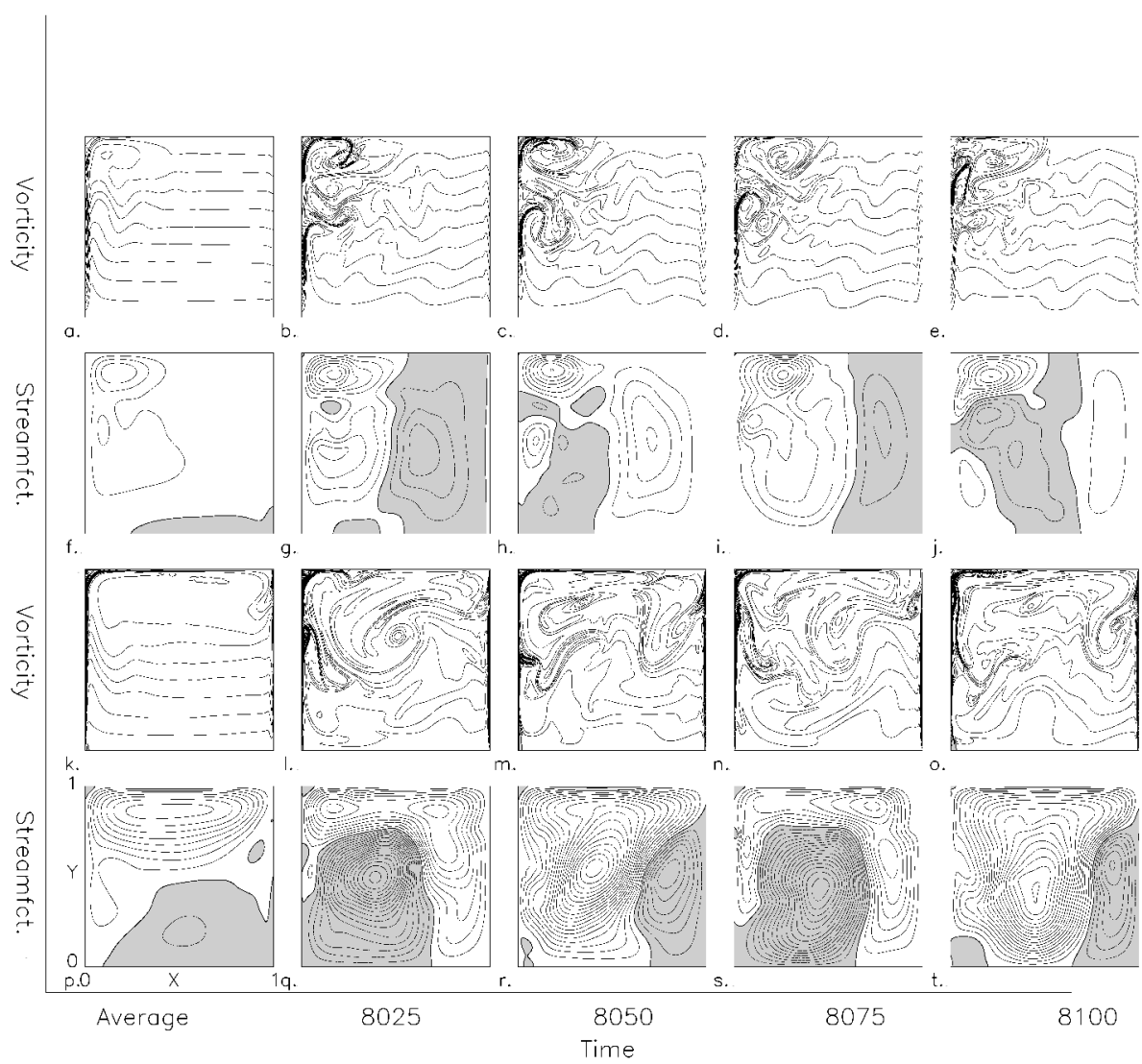

Figure 5. Snapshots and the time-mean of absolute vorticity and streamfunction are given for the $\operatorname{Re}_{\mathrm{b}}=0.25, \mathrm{Re}_{\mathrm{i}}=5$ and $\mathrm{Re}_{\mathrm{b}}=5, \mathrm{Re}_{\mathrm{i}}=5$ calculations. The means are in (a), (f), (k), and (p). The upper two rows show the $\operatorname{Re}_{\mathrm{b}}=0.25, \operatorname{Re}_{\mathrm{i}}=5$ calculation. The lower two rows show the $\operatorname{Re}_{\mathrm{b}}=5$, $\mathrm{Re}_{\mathrm{i}}=5$ calculation. Contours are 0.1 for vorticity and 0.5 for streamfunction. Regions of negative value are shaded.

\section{b. Mechanism of vorticity transport}

The transport of vorticity is critical to the control of the circulation strength because if vorticity pools or drains from within a particular streamline by an imbalance in supply and transport, the circulation around that streamline will change. Therefore, the transport of vorticity in each calculation has been diagnosed.

Ultimately, the friction must remove the vorticity from the basin but en route there are many possible pathways for transport of vorticity. The wind input generates primarily a time-mean planetary vorticity advection flux toward the western boundary: a nearly Sverdrup (1947) interior solution. The mean flow enters a boundary current as the vorticity flux is transformed to a mean advective flux (per Charney, 1955). Some of this boundary current enters the frictional region where its excess vorticity is removed. Much of the 

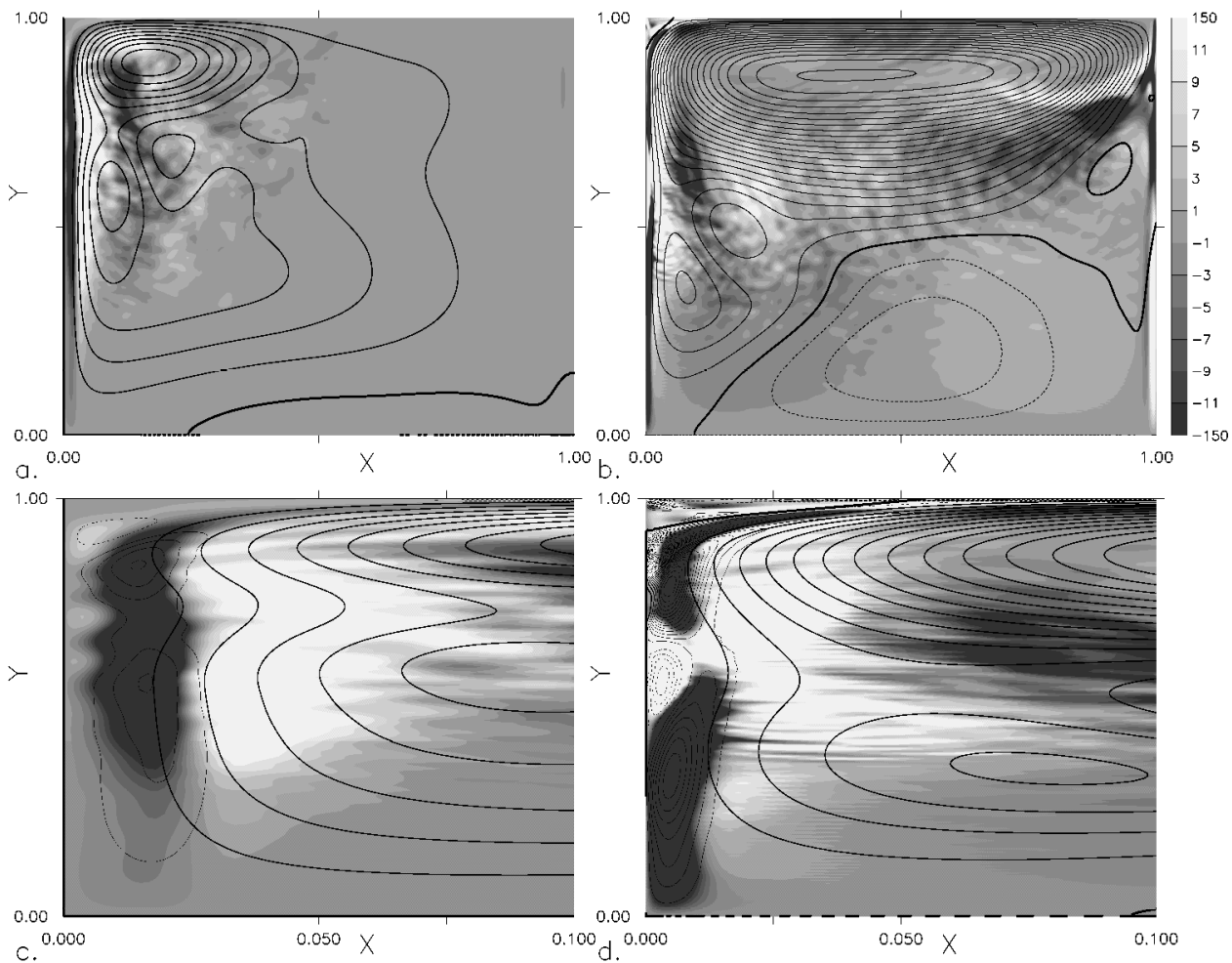

Figure 6. (a) The time-mean streamfunction contours (interval $=0.2$ ) are superimposed on the eddy flux convergence (shaded) for the $\mathrm{Re}_{\mathrm{b}}=0.25, \mathrm{Re}_{\mathrm{i}}=5$ calculation. Lighter shading denotes convergences of $\overline{\mathbf{u}^{\prime} \zeta^{\prime}}$; darker shading denotes divergences. (b) is similar to (a) but for $\operatorname{Re}_{\mathrm{b}}=5$, $\mathrm{Re}_{\mathrm{i}}=5$. (c) and (d) are close-ups near the western boundary of (a) and (b), respectively.In (c-d), the friction flux divergence is also superimposed with thin lines (interval $=10$, no zero contour, dashed/solid for divergence/convergence).

boundary current avoids this region, however. This causes the steady-state boundary current solution to no longer exist or become unstable at a certain point along the boundary, and the boundary current forms eddies. The eddies transport the vorticity across the mean streamlines to a frictional sublayer where it is removed from the basin by a frictional flux.

i. Flux convergences. Figure 6a shows the action of the frictional and eddy fluxes of vorticity on the mean flow for a western-intensified equilibrium. The contours of the mean flow and the eddy flux convergence are shown (the convergence is the negative of the flux divergence). The eddies interact with the mean flow in two primary regions, within the western boundary current and within the recirculation gyre.

Figure $6 \mathrm{c}$ is a close-up of Figure 6a, and it shows that within the western boundary current the effect of the eddies is to transport the wind vorticity input in the interior of the 
basin toward the western boundary. Once there, the frictional dissipation disposes of it. (Equivalently, one can consider the eddies to flux positive vorticity from the western boundary to the basin interior where it cancels the negative vorticity input from the wind.) The white region of eddy flux convergence near $x=0.05$ removes negative vorticity from the mean flow, and the black region of eddy flux divergence near $x=0.02$ deposits it closer to the boundary. The frictional contours above the eddy flux divergence in Figure $6 \mathrm{c}$ indicate that the friction receives the negative vorticity from the eddies and removes it from the basin. Thus, in the western boundary current the eddies widen the influence of the friction to the interior streamlines, in a manner reminiscent of eddies in traditional nonrotating turbulent boundary layers (e.g., Tennekes and Lumley, 1972).

Snapshots of the western-intensified solution shown in Figure $6 \mathrm{a}$ and $6 \mathrm{c}$ are shown in Figure $5 \mathrm{~b}-\mathrm{e}$ and Figure $5 \mathrm{~g}-\mathrm{j}$. These snapshots reveal both basin-scale eddy activity and smaller eddies in the western boundary current and recirculation gyre. As most of the eddy flux divergence in Figure 6a and 6c occurs in the western boundary current region, it is clear that the smaller eddies in that location transport more vorticity. However, the nonlinear effects of the larger modes are also important (see Section 6 of the companion paper (Fox-Kemper, 2004a) in this issue).

Curiously, the effect of eddies is to steepen the vorticity gradients in western-intensified calculations. The eddies flux vorticity up the gradient in the western boundary current, and are in that sense anti-frictional. This peculiar effect goes against the usual arguments for a frictional operator as an eddy parameterization. However, the sum of the frictional fluxes and the eddy fluxes is not up the gradient, or at least not to the same degree.

Within the recirculation gyre, the eddies are active while the frictional terms are less so. In this region the eddies transport the majority of the vorticity input across the mean streamlines. Eddies in the recirculation gyre also homogenize the absolute vorticity (per Rhines and Young, 1984). Figure 5a and 5k show smooth regions of time-mean absolute vorticity within the recirculation gyre.

In Figure $6 \mathrm{~b}$ and $6 \mathrm{~d}$, the flux convergences and mean flow are shown for a calculation which is inertially-dominated. The intense eddy flux convergence extending from the eastern boundary at approximately $y=0.85$ in Figure $6 \mathrm{~b}$ is important in regulating the strength of inertially-dominated equilibria. When the western frictional sublayer removes little of the wind input during spin-up, the recirculation continues to build up negative vorticity and enlarges until the recirculation reaches the eastern boundary. Once the eastern boundary is reached, the eddies are able to tear vorticity from both the eastern and western frictional sublayers and reaches equilibrium (Fig. 8). Because of the qualitative change in behavior when the recirculation reaches the eastern boundary, these calculations have different parametric dependence on Reynolds number than the western-intensified calculations (Fig. 4). The eastern boundary is needed because the northern boundary is not effective in helping to remove negative vorticity from the basin in these calculations (Fig. 8 , a result likely to change with different northern or eastern boundary conditions). 
ii. Removal crisis. There is a white region of eddy flux convergence within the frictional sublayer of the western boundary current ( $x$ near $0, y$ between 0.5 and 0.7 ) in Figure 6d that is not present in Figure $6 \mathrm{c}$. This region of convergence is consistent with the notion that the viscosity is insufficient in Figure 6d to remove all of the vorticity brought into the frictional sublayer by the mean flow and eddies. It indicates that the eddy flux of vorticity to the frictional sublayer reverses direction, transporting negative vorticity elsewhere. In contrast, the black regions in the frictional sublayer (Fig. 6c-d) import negative vorticity from eddy fluxes to be removed from the basin by friction. For this reason, we will call the appearance of this flux convergence the removal crisis.

In the removal crisis region in Figure 6d, negative vorticity is brought into the basin by friction, adding to the wind input of negative vorticity (Fig. 8b). The amount of vorticity brought into the basin must equal the wind input for an equilibrium to be reached, but if the frictional flux changes sign near this white region, the frictional flux elsewhere must be stronger for a basin-wide equilibrium to be reached. Thus, the relative vorticity gradients near the boundary increase. The increase in these gradients makes the equilibrium less and less like those at lower Reynolds number.

The removal of vorticity through the western boundary depends on both the viscosity and the gradient of the time-mean vorticity $\left(-\delta_{M}^{3} \nabla \bar{\zeta}\right)$. Apparently, with constant viscosity the gradient of the vorticity cannot be large enough to remove the vorticity from the basin at the western boundary alone, because if it becomes too large its other effects - an intensely inertial boundary current and a large recirculation gyre - take over the entire basin first.

The removal crisis region is present in all of the calculations shown in Figure 3 where the recirculation gyre reaches the eastern boundary. Once the recirculation gyre is large enough to reach the eastern boundary, vorticity can be fluxed into the basin from the eastern frictional sublayer as well as the western. This new source of vorticity causes a decrease in the kinetic energy response with increasing $\mathrm{Re}_{\mathrm{i}}$, as shown in Figure 4a.

The removal crisis may be the cause of the enlargement of the gyre, or the pattern of eddy flux convergence may be merely coincident with the new eddy interaction with the eastern boundary. It is generally difficult to distinguish the causality, as all of the events are linked in the inertially-dominated equilibrium. Whether a change in the eddy field, the recirculation gyre, or the boundary current initiates the process, all of these changes follow from one another. The total effect is that the eddy delivery of vorticity to the frictional sublayer and the frictional removal of vorticity through the western boundary layer is disrupted when the vorticity gradient becomes too large. However this removal crisis at the western boundary is caused, an equilibrium is reached by eventual enhancement of the rest of the western frictional sublayer and the eastern frictional sublayer.

iii. Meridional averages. The relative location of the peak of the meridionally-averaged eddy and mean flux convergences is different for the western-intensified and the inertiallydominated calculations, which is a sign of the removal crisis. Where the equilibrium is inertially-dominated, the mean flux divergence peaks closer to the boundary than the eddy 


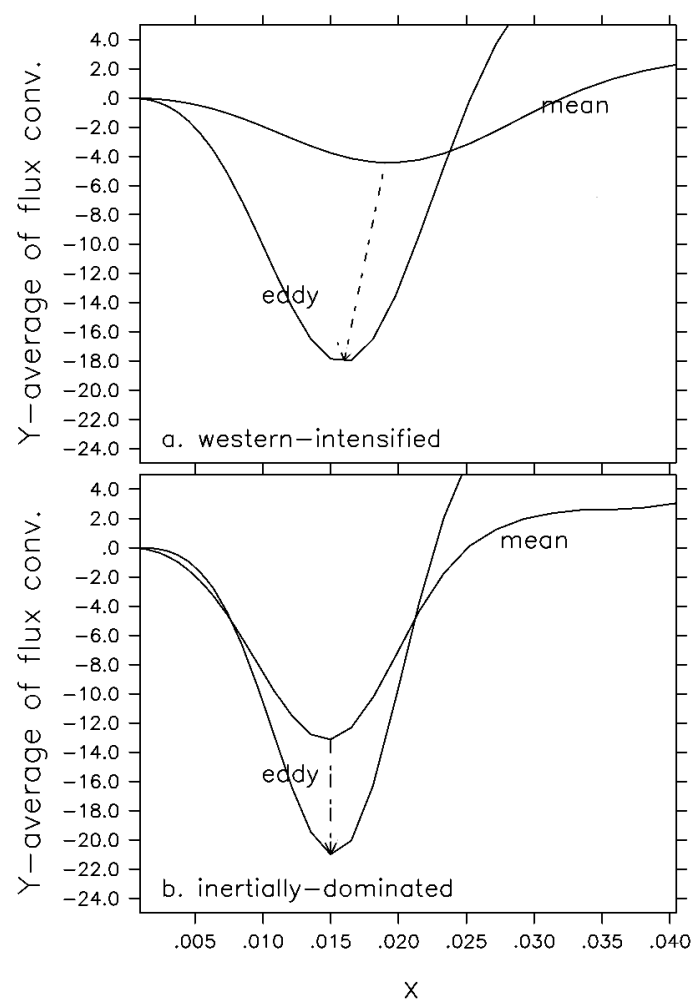

Figure 7. (a-b) show the average from $y=0$ to $y=1$ of the eddy and mean vorticity flux convergences as a function of $x$ within the frictional sublayer for two different calculations: (a) $\operatorname{Re}_{\mathrm{b}}=0.25, \mathrm{Re}_{\mathrm{i}}=5$ (western-intensified), and (b) $\mathrm{Re}_{\mathrm{b}}=0.25, \mathrm{Re}_{\mathrm{i}}=9$ (inertially-dominated). In the inertially-dominated equilibrium the mean advection delivers negative vorticity closer to the boundary than the eddy advection, while in the western-intensified case the eddies transport negative vorticity closer to the boundary.

flux divergence. In contrast, western-intensified calculations have the eddies delivering the vorticity deeper into the frictional sublayer. In a parameter range where (1) the steady-state calculation is inertially-dominated, but (2) the time-dependent calculation is westernintensified, it is sensible that the eddies should be able to deliver vorticity closer to the boundary than the mean flow. Otherwise, the eddies would be no more effective in reducing the circulation than the mean flow.

The relative location of the peaks therefore tests whether the eddies are able, on average, to deliver vorticity closer to the boundary than the mean flow. This test accurately predicts whether the flow will be inertially-dominated or not for calculations with $\mathrm{Re}_{\mathrm{i}}$ larger than 1 . In inertially-dominated calculations, the peak of meridionally-averaged eddy flux divergence is located farther from the boundary as a result of averaging over the region of flux convergence of the removal crisis. Figure 7 compares 

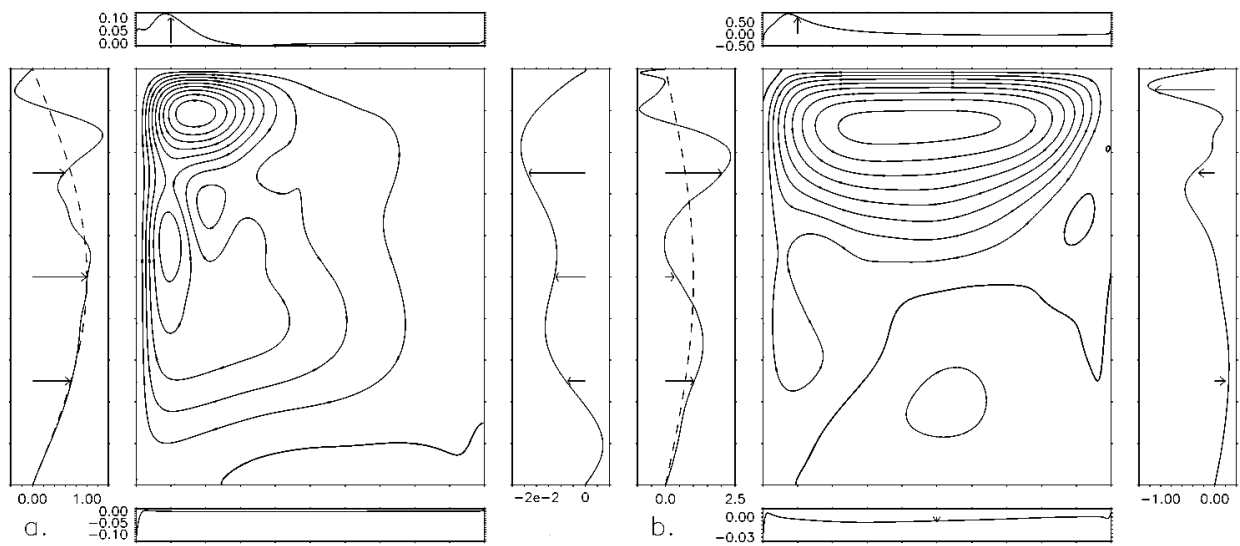

Figure 8. Maps of the normal frictional flux through each of the boundaries for (a) the westernintensified $\operatorname{Re}_{\mathrm{b}}=0.25, \operatorname{Re}_{\mathrm{i}}=5$ calculation and (b) the inertially-dominated $\operatorname{Re}_{\mathrm{b}}=5, \operatorname{Re}_{\mathrm{i}}=5$ calculation (on right). The four plots surrounding each contour plot indicate the frictional flux through the nearest boundary to each box $\left(-\delta_{M}^{3} \nabla \zeta\right)$ as a function of distance along the boundary. The flux through the western boundary needed to remove the wind vorticity input at the same latitude is overlaid with dashed lines. Arrows denote the direction of the frictional flux of positive vorticity. Note that the scales of the flux plots are different.

the location of the peaks in an inertially-dominated versus a western-intensified calculation.

iv. Fluxes through the boundary. Figure 8 shows the frictional flux through the boundary for a western-intensified and an inertially-dominated calculation. The removal crisis is present in the flux through the western boundary of the inertially-dominated calculation. A dip in the flux of the western-intensified calculation also occurs, but it does not reverse the direction of the flux. Note how the maximum flux through the western boundary is nearly 2.5 times larger in the inertially-dominated case than in the western-intensified one. Because the value of the viscosity at the boundary is 20 times smaller in the inertially-dominated case, this means that the maximum vorticity gradient at the boundary in this calculation is approximately 50 times greater than in the western intensified case! The fluxes through the eastern boundary are negligible in the western-intensified case, while in the inertially-dominated case the maximum flux through the eastern boundary is only two times smaller than the maximum through the western boundary. Thus, Figure 8 clearly shows the removal crisis, the larger gradient of vorticity at the boundary in the inertially-dominated case, and the necessity of the recirculation gyre reaching the eastern boundary in the inertially-dominatedcalculation.

The frictional flux through the western boundary needed to remove the wind input at the same latitude where it is injected (as in Stommel, 1948; Munk, 1950), and the primary assumption of Hughes and de Cuevas (2001) is indicated with dotted lines in Figure 8. Clearly, the dip in the frictional flux and the reversal of the frictional flux in the northwest corner make the removal of vorticity occur at a different latitude in the western-intensified 

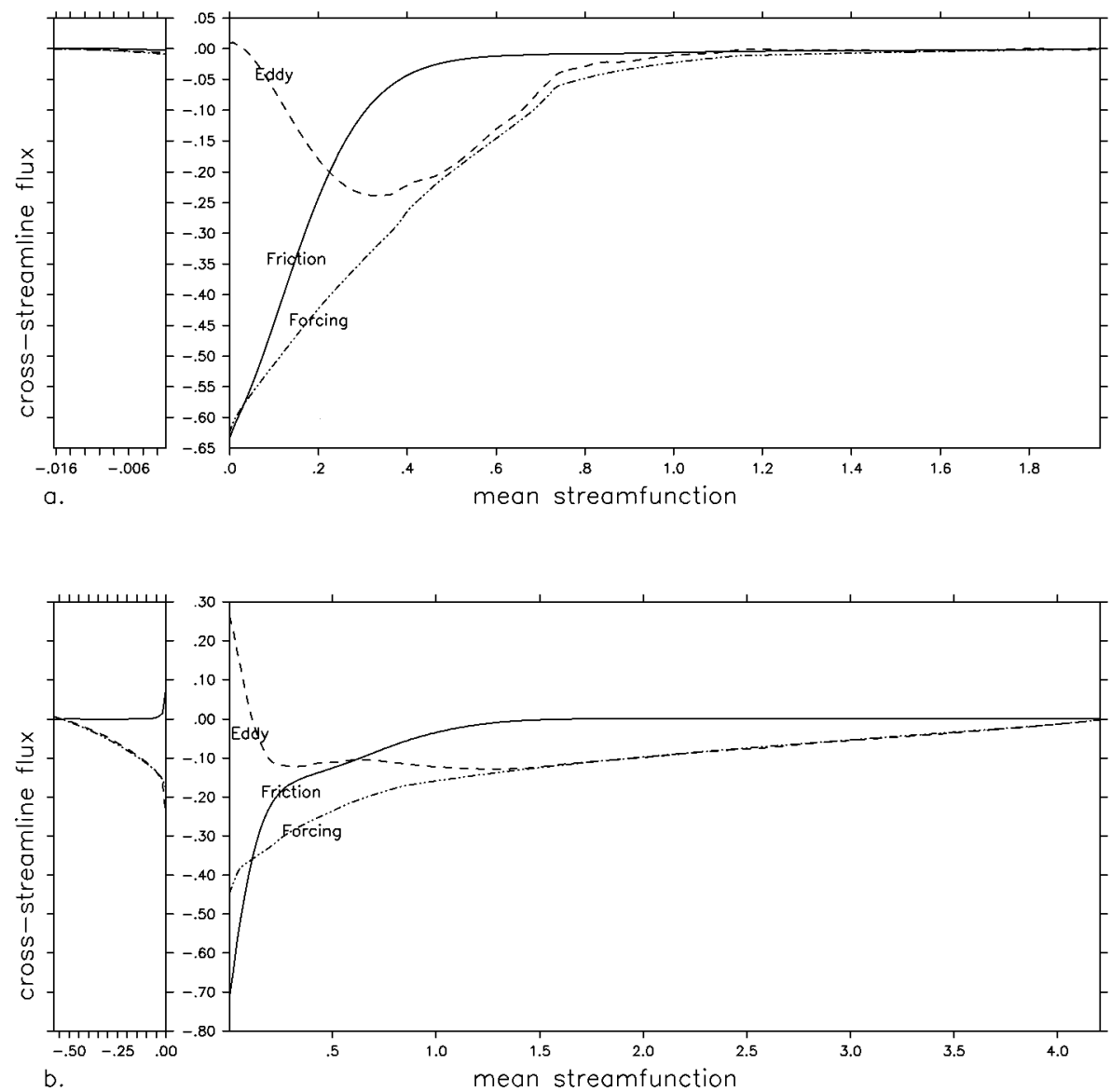

Figure 9. (a-b) show the vorticity flux across mean streamlines. Shown are the eddy flux out of the streamline $\left(\oint_{\overline{\psi_{c}}} \overline{\mathbf{u}^{\prime} \zeta^{\prime}} \cdot \hat{\mathbf{n}} \mathrm{ds}\right)$, the friction flux out of the streamline $\left(-\oint_{\bar{\psi}_{c}} \delta_{M}^{3} \nabla \bar{\zeta} \cdot \hat{\mathbf{n}} \mathrm{ds}\right)$, and the wind forcing within that streamline $\left(-\iint_{A\left(\bar{\psi}_{c}\right)} \sin (\pi y) \mathrm{dxdy}\right)$. A balanced budget results when the eddy flux plus the friction flux equals the forcing. (a) shows the result for the $\mathrm{Re}_{\mathrm{b}}=0.25, \mathrm{Re}_{\mathrm{i}}=5$ calculation. (b) shows the result for the $\mathrm{Re}_{\mathrm{b}}=5, \mathrm{Re}_{\mathrm{i}}=5$ calculation. The sum of the frictional fluxes at the boundary is the frictional removal from the basin. The sum of the eddy fluxes at the boundary is zero.

calculation. These effects are even more pronounced in the inertially-dominated calculation, where a significant portion of the frictional flux is through the eastern boundary. However, the "efficiency" of eddies in vorticity transport to the boundary can change this result dramatically (Fox-Kemper, 2004b).

v. Flux across mean streamlines. Figure 9a shows the total frictional and eddy fluxes through a mean streamline for the same calculation as shown in Figure 6a which is western-intensified (see Section 3 for conventions). For $\bar{\psi} \leq 0.2$, the eddy fluxes carry 


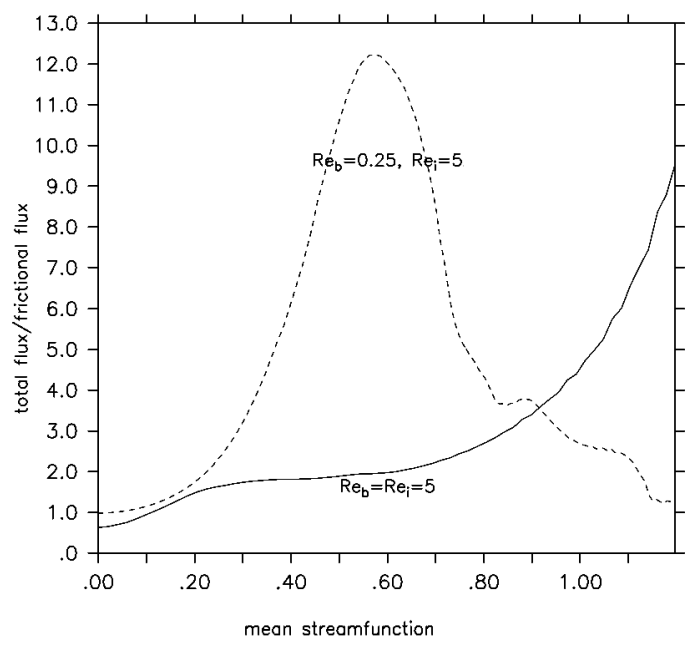

Figure 10. The flux ratio, $\mathrm{Nu}_{\zeta}$, for the $\mathrm{Re}_{\mathrm{b}}=\mathrm{Re}_{\mathrm{i}}=5$ calculation (solid) and the $\mathrm{Re}_{\mathrm{b}}=0.25, \mathrm{Re}_{\mathrm{i}}=5$ calculation (dashed).

more of the vorticity flux. So, in this western-intensified equilibrium, the wind input is carried from the interior streamlines to the region near the boundary (where $\bar{\psi} \approx 0$.2) by the eddies. Near the boundary, the eddies deposit the vorticity in the frictional region where it is removed from the basin.

Figure $9 \mathrm{~b}$ shows the frictional and eddy fluxes through a mean streamline for the same calculation as shown in Figure 6b which is inertially-dominated. In this calculation the maximum of the streamfunction is much larger, as the circulation is inertially dominated. However, even here the eddy flux of vorticity is larger than the frictional flux for $\bar{\psi}\ulcorner 0.5$. There is a large eddy flux even in the inertially-dominated calculation (some of which is from the eastern boundary), and the wind input within the innermost streamlines is removed almost entirely by eddy fluxes.

Although there are large eddy fluxes in the inertially-dominated case, the eddies in the western-intensified calculation are more efficient as defined by the Nusselt-like flux ratio, $\mathrm{Nu}_{\zeta}$, described in Section 3. It is plotted in Figure 10 for the same calculations shown in Figure 9 . Figure 10 shows that the calculation with boundary-enhanced viscosity has more of its total vorticity flux across mean streamlines near the boundary carried by the eddies in the basin interior. For both calculations, $\mathrm{Nu}_{\zeta}$ approaches one as $\bar{\psi}$ approaches zero as there is no eddy flux through the basin boundary. (Although the eddy flux across the zero mean streamline is not quite zero due to the flux into the region of negative streamfunction.) The eddies are able to control the circulation strength in the boundary-enhanced viscosity case while they are not able to do so in the constant viscosity case.

In summary, eddy fluxes are the primary transport of vorticity across mean streamlines in the basin interior. Only in the frictional sublayer is the frictional flux dominant. If the transfer of vorticity to the frictional fluxes is sufficient, the eddies are able to flux vorticity 
deeper into the sublayer than the mean flow can. This flux prevents inertial domination. Therefore, the eddies do replace the frictional flux across mean streamlines in the basin interior as the viscosity is lowered.

\section{Conclusion}

In this paper, the well-known result that the eddies in a wind-driven, single-gyre model are unable to accommodate a reduction in the viscosity everywhere in the basin is confirmed. The eddies are unable to control the circulation in this case because the eddies are capable of replacing the frictional fluxes across mean streamlines only in the basin interior. They are unable to ultimately remove vorticity through the basin boundaries.

Because the friction is intended in part to parameterize the effects of eddies, this result has caused much confusion in the past. It was thought that as the parametrization was removed, the newly energized eddies would be able to take over for the parameterization. Because this does not occur, the resulting domination of mean inertial terms throughout the basin is sometimes called inertial runaway. However, the friction in the homogeneous model represents not only the eddies, but also the unresolved interactions with the boundary. In particular, it represents all of the processes that might ultimately remove vorticity from the basin. By using a constant viscosity, one tacitly assumes that these processes are equivalent to the unresolved eddy processes. By using different viscosities to distinguish the parameterization of different physics, the circulation is controlled even with low interior viscosity.

The boundary-enhanced viscosity is intended to be a very simple parameterization of boundary processes, some of which might be described as viscous and some of which are not, such as bottom pressure torques. Of course, a more realistic parameterization would also be dynamically variable as well as spatially variable. Simplicity motivates the choice of only lateral friction with spatially-variable viscosity. Obviously, some aspects of the results found here will not apply when a more realistic treatment is possible. Even in this simple model, simultaneously resolving the boundary layer and calculating the entire basin circulation requires nontrivial computational power. However, the boundary-enhanced viscosity acts primarily as a sink of vorticity and a generator of eddies which are properties likely to be shared by a more realistic model, so it is hoped that many of the results presented here will be robust.

The removal of vorticity through the western boundary relies on both the viscosity and the gradient of the time-mean relative vorticity (the vorticity flux is $\delta_{M}^{3} \nabla \bar{\zeta}$ ). Thus, as the viscosity near the boundary decreases, the relative vorticity gradient increases. With small enough viscosity, the eddy vorticity transport to the frictional sublayer is interrupted by the removal crisis. When this occurs, the eddies do not deliver the vorticity any closer to the boundary than the mean flow does, and so the interior streamlines of the mean flow have no outlet for the vorticity input by the wind. Only when the recirculation gyre reaches the eastern boundary and a new source of vorticity is found is an equilibrium made possible.

As in the real ocean, although eddies in the interior may transport vorticity, they cannot create or destroy it. Therefore, there will always be a role for boundary processes in the 
vorticity budget in the ocean. However, it is not necessary to have a direct connection between the time-mean circulation and these boundary processes. Eddies are able to transport the vorticity from the mean flow to these boundary processes.

Finding a realistic physical mechanism by which the vorticity can be removed has been quite a challenge. The original models of Stommel (1948) and Munk (1950), and even many current numerical models, rely on overly large values of bottom drag and viscosity. Other detailed mechanisms are possible, such as bottom pressure torques as proposed by Hughes and de Cuevas (2001). Instead of focusing on the mechanism of the ultimate removal, this paper emphasizes the importance of the transport of vorticity to the region where the mechanism for ultimate removal is most active.

There is ample reason to believe that inertial domination will occur in more inclusive ocean models in the future. General circulation models cannot be operated with viscosities small enough for this to be a problem now, but this will be possible soon. Then, it will be time to turn not to the strengthening of eddy processes in the interior, but to the interaction of eddies and the boundary, or possibly to the interaction of eddies with neighboring gyres, a topic discussed in Fox-Kemper (2004b). The eddies produced in this model will have analogues in more complex models, and it seems likely that the additional types of instability that lead to eddies in more complex models (e.g., baroclinic instability) should make it easier for the eddies to perform the transport of vorticity across mean streamlines. Thus, while the eddy transport in general circulation models seems assured, ensuring that the boundaries are sufficiently capable of removing vorticity will also be important. It is now clear that the interaction of eddies with the boundary will play a role in correct prediction of the transport and recirculations of the western boundary currents.

Acknowledgment. B.F.-K. would like to thank his thesis advisers for their help and guidance. Thanks also to S. Jayne who contributed almost all of the computer resources. Very useful comments were provided by P. Berloff, G. Flierl, J. Franklin, M. Spall, V. Sheremet, and two anonymous reviewers. B.F.-K. was supported in part by an ONR-supported NDSEG Fellowship, an MIT Presidential Fellowship, a GFDL/Princeton University postdoctoral fellowship, and a NOAA Climate and Global Change postdoctoral fellowship (managed by UCAR). Both authors were supported in part by NSF OCE 9910654. This is Woods Hole Oceanographic Institution contribution number 10927.

\section{REFERENCES}

Berloff, P. 2004. Modeling the ocean circulation with reduced dynamics. Part I: Phenomenology of the eddy fluxes. J. Phys. Oceanogr. (submitted).

Bryan, K. 1963. A numerical investigation of a nonlinear model of a wind-driven ocean. J. Atmos. Sci., 20, 594-606.

Charney, J. G. 1955. The Gulf Stream as an inertial boundary layer. Proc. Nat. Acad. Sci., 41, 731-740.

Fox-Kemper, B. 2003. Friction and Eddies: Removal of Vorticity from the Wind-Driven Gyre. Ph.D. Thesis, MIT/WHOI, 2003-06,310 pp.

_ - 2004a. Wind-driven barotropic gyre II: Effects of eddies and low interior viscosity. J. Mar. Res., 62, 195-232.

_ $2004 \mathrm{~b}$. Re-evaluating the roles of eddies in multiple barotropic wind-driven gyres. J. Phys. Oceanogr. (submitted). 
Fox-Kemper, B., R. Ferrari and J. Pedlosky. 2003. On the indeterminacy of rotational and divergent eddy fluxes. J. Phys. Oceanogr., 33, 478-483.

Gottlieb, D. and S. A. Orszag. 1981. Numerical Analysis of Spectral Methods: Theory and Applications, Society for Industrial and Applied Mathematics, Philadelphia, PA, 170 pp.

Haynes, P. and M. McIntyre. 1987. On the evolution of vorticity and potential vorticity in the presence of diabatic heating and frictional or other forces. J. Atmos. Sci., 44, 828-841.

Holland, W. R. and L. B. Lin. 1975. On the origin of mesoscale eddies and their contribution to the general circulation of the ocean. II. A parameter study. J. Phys. Oceanogr., 5, 658-669.

Holland, W. R. and P. B. Rhines. 1980. An example of eddy-induced ocean circulation. J. Phys. Oceanogr., 10, 1010-1031.

Hughes, C. W. and B. A. de Cuevas. 2001. Why western boundary currents in realistic oceans are inviscid: A link between form stress and bottom pressure torques. J. Phys. Oceanogr., 31, 2871-2885.

Ierley, G. R. and O. G. Ruehr. 1986. Analytic and numerical solutions of a nonlinear boundary-layer problem. Studies in Appl. Math, 75, 1-36.

Ierley, G. R. and V. A. Sheremet. 1995. Multiple solutions and advection-dominated flows in the wind-driven circulation. Part I: Slip. J. Mar. Res., 53, 703-737.

Il' in, A. M. and V. M. Kamenkovich. 1964. The structure of the boundary layer in a two-dimensional model of ocean currents. Okeanologiya, 4, 756-769, in Russian.

Kamenkovich, V. M., V. A. Sheremet, A. R. Pashtushkov and S. Belotserkovsky. 1995. Analysis of the barotropic model of the subtropical gyre in the ocean for finite Reynolds numbers, Part I. J. Mar. Res., 53, 959-994.

LaCasce, J. and A. S. Bower. 2000. Relative dispersion in the subsurface North Atlantic. J. Mar. Res., 58, 863-894.

Marshall, J. C. 1984. Eddy mean flow interaction in a barotropic ocean model. Quart. J. Roy. Meteor. Soc., 100, 573-590.

Marshall, J. C. and G. Nurser. 1992. Fluid dynamics of oceanic thermocline ventilation. J. Phys. Oceanogr., 22, 1315-1329.

Munk, W. H. 1950. On the wind-driven ocean circulation. J. Meteor., 7, 79-93.

Niiler, P. P. 1966. On the theory of the wind-driven ocean circulation. Deep-Sea Res., 13, 597-606.

Pedlosky, J. 1987. Geophysical Fluid Dynamics, Springer, Berlin, 2nd edition, 710 pp.

—_ 1996. Ocean Circulation Theory, Springer, Berlin, 453 pp.

Rhines, P. B. and W. R. Young. 1984. Homogenization of potential vorticity in planetary gyres. J. Fluid Mech., 122, 347-367.

Scott, R. B. and D. N. Straub. 1998. Small viscosity behavior of a homogeneous, quasigeostrophic, ocean circulation model. J. Mar. Res., 56, 1225-1258.

Sheremet, V. A., G. R. Ierley and V. M. Kamenkovich. 1997. Eigenanalysis of the two-dimensional wind-driven ocean circulation problem. J. Mar. Res., 55, 57-92.

Sheremet, V. A., V. M. Kamenkovich and A. R. Pashtushkov. 1995. Analysis for the barotropic model of the subtropical gyre in the ocean for finite Reynolds numbers, Part II. J. Mar. Res., 53, 995-1024.

Stommel, H. M. 1948. The westward intensification of wind-driven ocean currents. Trans. Amer. Geophys. Union, 29, 202-206.

Sverdrup, H. U. 1947. Wind-driven currents in a baroclinic ocean; with application to the equatorial currents of the eastern Pacific. Proc. Nat. Acad. Sci., 33, 318-326.

Tennekes, H. and J. L. Lumley. 1972. A First Course in Turbulence, MIT Press, Cambridge, 300 pp.

Veronis, G. 1966. Wind-driven ocean circulation-Part II. Numerical solution of the nonlinear problem. Deep-Sea Res., 13, 30-55. 